\title{
Neural network application in forecasting maximum wall deflection in homogenous clay
}

\author{
Khalid R. Aljanabi ${ }^{1 *}$ (D) and Osamah M. AL-Azzawi
}

\section{${ }^{*}$ Correspondence:}

Kr_aljanabi@uoanbar.edu.iq

${ }^{1}$ Civil Engineering, University

of Anbar, Anbar, Iraq

Full list of author information

is available at the end of the

article

\begin{abstract}
An attempt was carried out by using a neural network to predict the maximum deflection and its position caused by braced excavation in homogeneous clay. Six input variables, including excavation depth, Ratio of El wall/El of brace, the vertical distance between bracing, Length to width ratio of an excavation, shear strength, and the coefficient of lateral earth pressure, were adopted. Two models were developed, one is to estimate the maximum deflection and the other one to estimate the position of maximum deflection. The ANN models were developed and verified using a database of (169) cases of actual measured and presumptive cases using the analysis with the Finite element of maximum deflection. A sensitivity analysis was accomplished, to examine the relative significance of the parameters that influence the maximum deflection of the wall and its position; it indicates that the Ratio of El wall/El of brace has the most significant effect on the maximum wall deflection, while the properties of the soil have the most considerable effects on the position. The results show that the ANN can reasonably forecast the magnitude of the maximum deflection of the wall, as well as its position. Design charts are developed based on the ANN model.
\end{abstract}

\section{Highlights}

- Neural Networks was used to forecast maximum deflection of braced excavation in homogeneous clay and its position.

- A sensitivity analysis was accomplished to examine the relative significance of the parameters that influence the models.

- The results confirm that the developed ANN model is able to predict maximum deflection and its position reliably.

- Design charts were developed based on the ANN model.

Keywords: Maximum wall deflection, Braced excavation, Homogeneous clay, Neural network, Forecasting author(s) and the source, provide a link to the Creative Commons licence, and indicate if changes were made. The images or other third party material in this article are included in the article's Creative Commons licence, unless indicated otherwise in a credit line to the material. If material is not included in the article's Creative Commons licence and your intended use is not permitted by statutory regulation or exceeds the permitted use, you will need to obtain permission directly from the copyright holder. To view a copy of this licence, visit http:// creativecommons.org/licenses/by/4.0/. 


\section{Introduction}

Geotechnical engineers facing complicated multivariate problems, which include several interacting factors. Generally, the relationship among these factors is not exactly known. The extraction of information to develop a relationship between these factors required advanced techniques for modeling beside a human experience. Braced excavation is popular in construction such as high multistoried buildings with basements, underground structures for transportation stations, and parking.

A safe excavation can be achieved using a satisfactorily braced wall; the braced wall also reduces deformations in the surrounding ground due to excessive wall deflections, which are most important to avoid costly damages to neighboring structures and buildings. In deep excavation projects, observational methods are involved to ensure safe construction, as well as used a finite element to forecast deflections of the wall in deep excavations $[1,15,16]$.

Some design charts were established for predicting the maximum lateral deflection of the wall based on depth of excavation, support system, and wall length [7, 9] see Fig. 1.

Artificial neural networks (ANNs) form a category of frameworks, which are mimicked neural networks in living creatures. An important merit of ANNs is learning, learning models, (ANNs) can deal with incomplete, qualitative, and uncertain data effectively. Therefore, for modeling sophisticated problems in which the equations that govern the problems are difficult to set, ANN is a highly promising tool. ANNs can be performed in mapping, transitory and optimization problems, besides modeling dynamic processes [3]. Generally, the number of input parameters used should be small in artificial neural networks to predict the maximum lateral deflection of the wall in clays with soft to medium consistency. The database used to develop the ANN is generated from presumptive cases employing the method of finite element (FEM) [11] and case histories of deep excavations for training, testing, and verification [8]. The objective of this work is

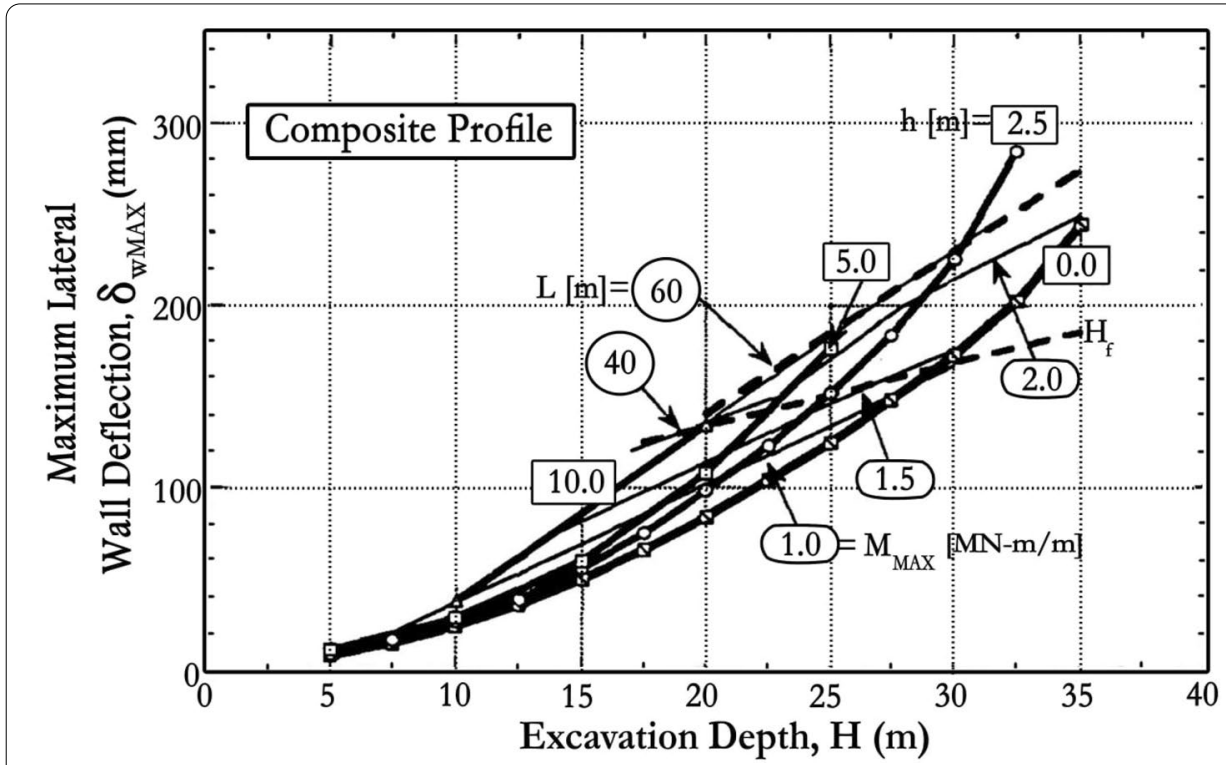

Fig. 1 design chart obtained by Hashash and Whittle [7] 
to employ (ANNs) to build a model to forecast maximum lateral wall deflection and its position in homogenous clays.

\section{Methodology}

\section{Artificial neural networks}

Artificial neural networks (ANNs) are simulated the nerve cells in the brain, they are considered as computational devices. Despite the complexity of the brain that cannot be approached by ANNs, there are two kinds of similarities. First, the computational units of networks are simple and interconnected to a high degree. The second one is that the connections among neurons set the function and feature of the network [5].

By adjusting weights among neurons, ANNs may be trained to perform a selected function. Neural networks are trained to perform complicated functions in extraordinary fields of application such as identification, pattern recognition, speech, vision, classification, and control systems.

Various tools have been used by engineers to perform informal modeling (mapping from cause to effect for estimation and prediction) and inverse mapping (mapping from effects to causes) which include knowledge-based systems, optimization, regression, statistics, probability, and others. Thus, ANN can be considered as another tool for engineers to perform either the modeling or inverse mapping [10]. Neural networks are applied in different fields of geotechnical engineering including parameter assessment, underground openings, foundations, site investigation, liquefaction, retaining structures, slopes, and ground movement [17].

\section{Back-propagations}

One of the neural networks is back-propagation which is vastly used in solving problems that need modeling nonlinear relationships. By modifying the weights so the error among the actual target and the desired target is minimized, as a result, the simulation of the networks will produce the output units, and the forecasting of the neural networks to the outputs is highly more correct.

When the internal parameters of the network have been corrected, the mechanism of correction begins with the output layers then the error back-propagates backward through each internal (hidden) layer. Hence, for this kind of network, the backpropagation is used [2].

\section{Levenberg Marquardt algorithm.}

The algorithm is based on the newton method variation in which the functions which are the sum of squares of other nonlinear functions are minimized, it is appropriate when MSE (Mean Square Error) is the index of performance.

The basic step of the Levenberg-Marquardt algorithm is:

$$
X_{K+1}=X_{K}-\left[J^{T} J+\mu I\right]^{-1} J^{T} e
$$

where (e) is a vector of network errors, (J) is the Jacobian matrix, (I) is a unit matrix, and $(\mu)$ is a positive scalar. The algorithm becomes gradient descent with a small step size when $\mu$ is large. For some input/output patterns, the Levenberg-Marquardt algorithm is very fast and effective compared to the standard gradient algorithms. Thus, in this 
study, the training algorithm used is the Levenberg-Marquardt algorithm with the mean square of errors as a performance function.

\section{Prediction of maximum wall deflection and it's position by ANN Development of ANN Models}

The database includes a total of (169) cases recorded from site observations and finite element analysis for homogeneous clayey soil types. The six parameters used as the ANN model inputs have the most considerable effect on the deep excavations in homogeneous clayey soil. Parameters include: Excavation depth $(\mathrm{H}) \mathrm{m}$, Ratio of Length of wall/width of excavation (L/B), ratio of EI of the wall / EI of the brace, the vertical distance of bracing $\left(h_{s}\right) \mathrm{m}$, undrained shear strength of soil $\left(\mathrm{Su}, \mathrm{kN} / \mathrm{m}^{2}\right)$, and the earth pressure coefficient of at rest (Ko). Table 1 shows the dataset used and its sources.

In this work, a computer program MATLAB (2015) Neural Network Toolbox is used for modeling the neural network. A summary of the basic characteristics of the ANN model utilized in this study is as follows: Type of network: Feed-forward backpropagation, Algorithm of training: (TRAINLM) Levenberg-Marquardt algorithm, the function of Learning: (LEARNGDM) Gradient descent with momentum, the function of performance: (MSE) Mean square error and the coefficient of correlation (R), the first layer transfer function: Sigmoid, the second transfer function: tangent-sigmoid.

The functions of the Sigmoid (logsig) and the hyperbolic tangent-sigmoid (tansig) can be expressed as in Eqs. 2 and 3 respectively:

$$
\begin{aligned}
& \operatorname{logsig}(n)=1 /(1+\exp (-n)) \\
& \operatorname{Tansig}(n)=[2 /(1+\exp (-2 n))]-1
\end{aligned}
$$

where: $\mathrm{n}$ is any value between $(-\infty)$ and $(+\infty)$. Figure 2 shows the details of (ANN).

\section{Data division and pre-processing}

The data set was randomly divided into three sets through the nntools box using the default percentages. The percentage of the training, testing, and validation are, 70\%, $15 \%$ and $15 \%$ respectively. Before building the neural network, the data Pre-processing is essential to put them into proportional form, which is carried out by scaling (normalizing) them so that they fall into a standard range -1 to 1 . In the output layer, the variables have to be normalized to be proportional to the transfer function limits that used in the output layer [5].

Scaling the data is important to assure that during the training, all the variables will receive equal attention. The scaled value $X_{n}$ is calculated as follows:

$$
X_{n}=\frac{2 \times\left(X-X_{\min }\right)}{\left(X_{\max }-X_{\min }\right)}-1
$$

\section{Results and discussion}

Two models were built one for maximum wall deflection and the other to the position of the maximum deflection, each model has six nodes that represent the inputs used and one node for the output required. 
Table 1 Data used in building the neural networks

\begin{tabular}{|c|c|c|c|c|c|c|c|c|}
\hline \multirow[t]{2}{*}{ References } & \multicolumn{6}{|c|}{ Input variables } & \multicolumn{2}{|l|}{ Output } \\
\hline & $\begin{array}{l}\text { Excavation } \\
\text { Depth, H } \\
(\mathrm{m})\end{array}$ & $\begin{array}{l}\text { Length } \\
\text { to width } \\
\text { ratio of } \\
\text { excavation } \\
\text { L/B }\end{array}$ & $\begin{array}{l}\text { Ratio of } \\
\text { El wall/ } \\
\text { El of } \\
\text { brace }\end{array}$ & $\begin{array}{l}\text { Vertical } \\
\text { Spacing } \\
\text { between } \\
\text { bracing, S } \\
\text { (m) }\end{array}$ & $\begin{array}{l}\text { Undrained } \\
\text { Shear } \\
\text { strength } \\
\text { of clay, Su } \\
\left(\mathrm{kN} / \mathrm{m}^{2}\right)\end{array}$ & $\begin{array}{l}\text { Coefficient } \\
\text { of earth } \\
\text { pressure at } \\
\text { rest, Ko }\end{array}$ & $\begin{array}{l}\text { Maximum } \\
\text { deflection, } \\
\delta_{H}(\mathrm{~mm})\end{array}$ & $\begin{array}{l}\text { Position of } \\
\text { maximum } \\
\text { deflection, } \\
\text { (m) }\end{array}$ \\
\hline \multirow{9}{*}{$\begin{array}{l}\text { Lam et al. } \\
\text { [12] }\end{array}$} & 2.16 & 0.204 & 0.108 & 1 & 25.5 & 0.53 & 92 & 3 \\
\hline & 4.32 & 0.204 & 0.108 & 2 & 25.5 & 0.53 & 78 & 5.6 \\
\hline & 5.15 & 0.204 & 0.108 & 3 & 25.5 & 0.53 & 38 & 6 \\
\hline & 2.16 & 0.204 & 2.808 & 1 & 25.5 & 0.53 & 34 & 9 \\
\hline & 4.32 & 0.204 & 2.808 & 2 & 25.5 & 0.53 & 12 & 9 \\
\hline & 5.15 & 0.204 & 2.808 & 3 & 25.5 & 0.53 & 7 & 9 \\
\hline & 2.16 & 0.204 & 8.509 & 1 & 25.5 & 0.53 & 90 & 8 \\
\hline & 4.32 & 0.204 & 8.509 & 2 & 25.5 & 0.53 & 23 & 8 \\
\hline & 5.15 & 0.204 & 8.509 & 3 & 25.5 & 0.53 & 3 & 8 \\
\hline \multirow{12}{*}{$\begin{array}{l}\text { Hashash } \\
\text { et al. [6] }\end{array}$} & 2.5 & 1 & 3.117 & 1 & 15 & 0.53 & 10 & 0 \\
\hline & 5 & 1 & 3.117 & 2 & 15 & 0.53 & 15 & 12 \\
\hline & 7.5 & 1 & 3.117 & 3 & 15 & 0.53 & 28 & 15 \\
\hline & 10 & 1 & 3.117 & 4 & 15 & 0.53 & 45 & 17.5 \\
\hline & 12.5 & 1 & 3.117 & 5 & 15 & 0.53 & 62 & 20 \\
\hline & 15 & 1 & 3.117 & 6 & 15 & 0.53 & 84 & 22.5 \\
\hline & 2.5 & 1 & 3.229 & 1 & 15 & 0.59 & 47 & 20 \\
\hline & 5 & 1 & 3.229 & 2 & 15 & 0.59 & 37 & 20 \\
\hline & 7.5 & 1 & 3.229 & 3 & 15 & 0.59 & 27 & 17.5 \\
\hline & 10 & 1 & 3.229 & 4 & 15 & 0.59 & 16 & 15 \\
\hline & 12.5 & 1 & 3.229 & 5 & 15 & 0.59 & 11 & 10 \\
\hline & 15 & 1 & 3.229 & 6 & 15 & 0.59 & 8 & 0 \\
\hline \multirow{3}{*}{$\begin{array}{c}\text { Nicholson } \\
{[14]}\end{array}$} & 2.6 & 0.15 & 0.796 & 2.5 & 16 & 0.6 & 1 & 70 \\
\hline & 7.6 & 0.15 & 0.796 & 2.5 & 16 & 0.6 & 8 & 80 \\
\hline & 14.7 & 0.15 & 0.796 & 2.5 & 16 & 0.6 & 10.5 & 95 \\
\hline \multirow[t]{4}{*}{ Lam [13] } & 17.5 & 1.333 & 3.229 & 2.5 & 80 & 0.53 & 100 & 25 \\
\hline & 17.5 & 1 & 3.229 & 2.5 & 80 & 0.53 & 110 & 25 \\
\hline & 17.5 & 0.8 & 3.229 & 2.5 & 80 & 0.53 & 120 & 25 \\
\hline & 17.5 & 0.667 & 3.229 & 2.5 & 80 & 0.53 & 130 & 25 \\
\hline \multirow[t]{18}{*}{ Jen [9] } & 5 & 0.625 & 3.229 & 2.5 & 80 & 0.53 & 17 & 10 \\
\hline & 5 & 0.625 & 12.915 & 2.5 & 80 & 0.53 & 17 & 10 \\
\hline & 5 & 0.625 & 19.355 & 2.5 & 80 & 0.53 & 17 & 10 \\
\hline & 5 & 0.625 & 25.806 & 2.5 & 80 & 0.53 & 17 & 10 \\
\hline & 5 & 0.625 & 32.287 & 2.5 & 80 & 0.53 & 17 & 10 \\
\hline & 5 & 0.625 & 38.71 & 2.5 & 80 & 0.53 & 17 & 10 \\
\hline & 7.5 & 0.625 & 6.455 & 2.5 & 80 & 0.53 & 35 & 14 \\
\hline & 7.5 & 0.625 & 12.915 & 2.5 & 80 & 0.53 & 38 & 14 \\
\hline & 7.5 & 0.625 & 19.355 & 2.5 & 80 & 0.53 & 38 & 14 \\
\hline & 7.5 & 0.625 & 25.806 & 2.5 & 80 & 0.53 & 38 & 14 \\
\hline & 7.5 & 0.625 & 32.287 & 2.5 & 80 & 0.53 & 38 & 14 \\
\hline & 7.5 & 0.625 & 38.71 & 2.5 & 80 & 0.53 & 40 & 14 \\
\hline & 10 & 0.625 & 6.455 & 2.5 & 80 & 0.53 & 50 & 16 \\
\hline & 10 & 0.625 & 12.915 & 2.5 & 80 & 0.53 & 55 & 16 \\
\hline & 10 & 0.625 & 19.355 & 2.5 & 80 & 0.53 & 56 & 16 \\
\hline & 10 & 0.625 & 25.806 & 2.5 & 80 & 0.53 & 57 & 16 \\
\hline & 10 & 0.625 & 32.287 & 2.5 & 80 & 0.53 & 58 & 16 \\
\hline & 10 & 0.625 & 38.71 & 2.5 & 80 & 0.53 & 60 & 16 \\
\hline
\end{tabular}


Table 1 (continued)

\begin{tabular}{|c|c|c|c|c|c|c|c|c|}
\hline \multirow[t]{2}{*}{ References } & \multicolumn{6}{|c|}{ Input variables } & \multicolumn{2}{|l|}{ Output } \\
\hline & $\begin{array}{l}\text { Excavation } \\
\text { Depth, H } \\
\text { (m) }\end{array}$ & $\begin{array}{l}\text { Length } \\
\text { to width } \\
\text { ratio of } \\
\text { excavation } \\
\text { L/B }\end{array}$ & $\begin{array}{l}\text { Ratio of } \\
\text { El wall/ } \\
\text { El of } \\
\text { brace }\end{array}$ & $\begin{array}{l}\text { Vertical } \\
\text { Spacing } \\
\text { between } \\
\text { bracing, S } \\
\text { (m) }\end{array}$ & $\begin{array}{l}\text { Undrained } \\
\text { Shear } \\
\text { strength } \\
\text { of clay, Su } \\
\left(\mathrm{kN} / \mathrm{m}^{2}\right)\end{array}$ & $\begin{array}{l}\text { Coefficient } \\
\text { of earth } \\
\text { pressure at } \\
\text { rest, Ko }\end{array}$ & $\begin{array}{l}\text { Maximum } \\
\text { deflection, } \\
\delta_{\mathrm{H}}(\mathrm{mm})\end{array}$ & $\begin{array}{l}\text { Position of } \\
\text { maximum } \\
\text { deflection, } \\
\text { (m) }\end{array}$ \\
\hline & 12.5 & 0.625 & 6.455 & 2.5 & 80 & 0.53 & 79 & 20 \\
\hline & 12.5 & 0.625 & 12.915 & 2.5 & 80 & 0.53 & 84 & 20 \\
\hline & 12.5 & 0.625 & 19.355 & 2.5 & 80 & 0.53 & 86 & 20 \\
\hline & 12.5 & 0.625 & 25.806 & 2.5 & 80 & 0.53 & 89 & 20 \\
\hline & 12.5 & 0.625 & 32.287 & 2.5 & 80 & 0.53 & 92 & 20 \\
\hline & 12.5 & 0.625 & 38.71 & 2.5 & 80 & 0.53 & 97 & 20 \\
\hline & 15 & 0.625 & 6.455 & 2.5 & 80 & 0.53 & 150 & 25 \\
\hline & 15 & 0.625 & 12.915 & 2.5 & 80 & 0.53 & 200 & 25 \\
\hline & 17.5 & 0.625 & 6.455 & 2.5 & 80 & 0.53 & 300 & 25 \\
\hline & 2.5 & 0.625 & 3.229 & 0 & 80 & 0.53 & 17 & 0 \\
\hline & 2.5 & 0.75 & 3.229 & 0 & 80 & 0.53 & 17 & 0 \\
\hline & 2.5 & 0.875 & 3.229 & 0 & 80 & 0.53 & 17 & 0 \\
\hline & 2.5 & 1 & 3.229 & 0 & 80 & 0.53 & 17 & 0 \\
\hline & 5 & 0.625 & 3.229 & 2.5 & 80 & 0.53 & 20 & 15 \\
\hline & 5 & 0.75 & 3.229 & 2.5 & 80 & 0.53 & 20 & 15 \\
\hline & 5 & 0.875 & 3.229 & 2.5 & 80 & 0.53 & 20 & 15 \\
\hline & 5 & 1 & 3.229 & 2.5 & 80 & 0.53 & 20 & 15 \\
\hline & 7.5 & 0.625 & 3.229 & 2.5 & 80 & 0.53 & 39 & 18 \\
\hline & 7.5 & 0.75 & 3.229 & 2.5 & 80 & 0.53 & 39 & 18 \\
\hline & 7.5 & 0.875 & 3.229 & 2.5 & 80 & 0.53 & 39 & 18 \\
\hline & 7.5 & 1 & 3.229 & 2.5 & 80 & 0.53 & 39 & 18 \\
\hline & 10 & 0.625 & 3.229 & 2.5 & 80 & 0.53 & 60 & 22.5 \\
\hline & 10 & 0.75 & 3.229 & 2.5 & 80 & 0.53 & 55 & 22.5 \\
\hline & 10 & 0.875 & 3.229 & 2.5 & 80 & 0.53 & 55 & 22.5 \\
\hline & 10 & 1 & 3.229 & 2.5 & 80 & 0.53 & 55 & 22.5 \\
\hline & 12.5 & 0.625 & 3.229 & 2.5 & 80 & 0.53 & 95 & 25 \\
\hline & 12.5 & 0.75 & 3.229 & 2.5 & 80 & 0.53 & 80 & 23 \\
\hline & 12.5 & 0.875 & 3.229 & 2.5 & 80 & 0.53 & 80 & 23 \\
\hline & 12.5 & 1 & 3.229 & 2.5 & 80 & 0.53 & 80 & 23 \\
\hline & 15 & 0.625 & 3.229 & 2.5 & 80 & 0.53 & 150 & 25 \\
\hline & 15 & 0.75 & 3.229 & 2.5 & 80 & 0.53 & 120 & 25 \\
\hline & 15 & 0.875 & 3.229 & 2.5 & 80 & 0.53 & 110 & 25 \\
\hline & 15 & 1 & 3.229 & 2.5 & 80 & 0.53 & 110 & 25 \\
\hline & 17.5 & 0.625 & 3.229 & 2.5 & 80 & 0.53 & 210 & 25 \\
\hline & 17.5 & 0.75 & 3.229 & 2.5 & 80 & 0.53 & 185 & 30 \\
\hline & 17.5 & 0.875 & 3.229 & 2.5 & 80 & 0.53 & 150 & 27 \\
\hline & 17.5 & 1 & 3.229 & 2.5 & 80 & 0.53 & 14.5 & 27 \\
\hline & 17.5 & 2.667 & 3.229 & 2.5 & 80 & 0.53 & 70 & 25 \\
\hline & 17.5 & 2 & 3.229 & 2.5 & 80 & 0.53 & 80 & 25 \\
\hline & 17.5 & 1.333 & 3.229 & 2.5 & 80 & 0.53 & 95 & 27 \\
\hline & 17.5 & 1 & 3.229 & 2.5 & 80 & 0.53 & 105 & 27 \\
\hline & 17.5 & 0.8 & 3.229 & 2.5 & 80 & 0.53 & 115 & 27 \\
\hline & 17.5 & 0.667 & 3.229 & 2.5 & 80 & 0.53 & 125 & 27 \\
\hline & 17.5 & 0.571 & 3.229 & 2.5 & 80 & 0.53 & 135 & 27 \\
\hline & 17.5 & 0.5 & 3.229 & 2.5 & 80 & 0.53 & 145 & 27 \\
\hline & 2.5 & 0.313 & 3.229 & 0 & 100 & 0.56 & 10 & 0 \\
\hline & 2.5 & 0.375 & 3.229 & 0 & 100 & 0.56 & 10 & 0 \\
\hline & 2.5 & 0.438 & 3.229 & 0 & 100 & 0.56 & 10 & 0 \\
\hline
\end{tabular}


Table 1 (continued)

\begin{tabular}{|c|c|c|c|c|c|c|c|c|}
\hline \multirow[t]{2}{*}{ References } & \multicolumn{6}{|c|}{ Input variables } & \multicolumn{2}{|l|}{ Output } \\
\hline & $\begin{array}{l}\text { Excavation } \\
\text { Depth, H } \\
\text { (m) }\end{array}$ & $\begin{array}{l}\text { Length } \\
\text { to width } \\
\text { ratio of } \\
\text { excavation } \\
\text { L/B }\end{array}$ & $\begin{array}{l}\text { Ratio of } \\
\text { El wall/ } \\
\text { El of } \\
\text { brace }\end{array}$ & $\begin{array}{l}\text { Vertical } \\
\text { Spacing } \\
\text { between } \\
\text { bracing, S } \\
\text { (m) }\end{array}$ & $\begin{array}{l}\text { Undrained } \\
\text { Shear } \\
\text { strength } \\
\text { of clay, Su } \\
\left(\mathrm{kN} / \mathrm{m}^{2}\right)\end{array}$ & $\begin{array}{l}\text { Coefficient } \\
\text { of earth } \\
\text { pressure at } \\
\text { rest, Ko }\end{array}$ & $\begin{array}{l}\text { Maximum } \\
\text { deflection, } \\
\delta_{\mathrm{H}}(\mathrm{mm})\end{array}$ & $\begin{array}{l}\text { Position of } \\
\text { maximum } \\
\text { deflection, } \\
\text { (m) }\end{array}$ \\
\hline & 2.5 & 0.5 & 3.229 & 0 & 100 & 0.56 & 10 & 0 \\
\hline & 5 & 0.313 & 3.229 & 2.5 & 100 & 0.56 & 15 & 12 \\
\hline & 5 & 0.375 & 3.229 & 2.5 & 100 & 0.56 & 15 & 12 \\
\hline & 5 & 0.438 & 3.229 & 2.5 & 100 & 0.56 & 15 & 12 \\
\hline & 5 & 0.5 & 3.229 & 2.5 & 100 & 0.56 & 15 & 12 \\
\hline & 7.5 & 0.313 & 3.229 & 2.5 & 100 & 0.56 & 30 & 17 \\
\hline & 7.5 & 0.375 & 3.229 & 2.5 & 100 & 0.56 & 30 & 17 \\
\hline & 7.5 & 0.438 & 3.229 & 2.5 & 100 & 0.56 & 30 & 17 \\
\hline & 7.5 & 0.5 & 3.229 & 2.5 & 100 & 0.56 & 30 & 17 \\
\hline & 10 & 0.313 & 3.229 & 2.5 & 100 & 0.56 & 45 & 20 \\
\hline & 10 & 0.375 & 3.229 & 2.5 & 100 & 0.56 & 45 & 20 \\
\hline & 10 & 0.438 & 3.229 & 2.5 & 100 & 0.56 & 45 & 20 \\
\hline & 10 & 0.5 & 3.229 & 2.5 & 100 & 0.56 & 45 & 20 \\
\hline & 12.5 & 0.313 & 3.229 & 2.5 & 100 & 0.56 & 65 & 25 \\
\hline & 12.5 & 0.375 & 3.229 & 2.5 & 100 & 0.56 & 60 & 20 \\
\hline & 12.5 & 0.438 & 3.229 & 2.5 & 100 & 0.56 & 60 & 20 \\
\hline & 12.5 & 0.5 & 3.229 & 2.5 & 100 & 0.56 & 60 & 20 \\
\hline & 15 & 0.313 & 3.229 & 2.5 & 100 & 0.56 & 95 & 25 \\
\hline & 15 & 0.375 & 3.229 & 2.5 & 100 & 0.56 & 80 & 24 \\
\hline & 15 & 0.438 & 3.229 & 2.5 & 100 & 0.56 & 80 & 24 \\
\hline & 15 & 0.5 & 3.229 & 2.5 & 100 & 0.56 & 80 & 24 \\
\hline & 17.5 & 0.313 & 3.229 & 2.5 & 100 & 0.56 & 127 & 25 \\
\hline & 17.5 & 0.375 & 3.229 & 2.5 & 100 & 0.56 & 108 & 30 \\
\hline & 17.5 & 0.438 & 3.229 & 2.5 & 100 & 0.56 & 102 & 27 \\
\hline & 17.5 & 0.5 & 3.229 & 2.5 & 100 & 0.56 & 102 & 27 \\
\hline & 20 & 0.313 & 3.229 & 2.5 & 100 & 0.56 & 152 & 25 \\
\hline & 20 & 0.375 & 3.229 & 2.5 & 100 & 0.56 & 14.6 & 30 \\
\hline & 20 & 0.438 & 3.229 & 2.5 & 100 & 0.56 & 126 & 30 \\
\hline & 20 & 0.5 & 3.229 & 2.5 & 100 & 0.56 & 124 & 30 \\
\hline & 17.5 & 0.313 & 3.229 & 2.5 & 110 & 0.58 & 105 & 25 \\
\hline & 17.5 & 0.375 & 3.229 & 2.5 & 110 & 0.58 & 87 & 30 \\
\hline & 17.5 & 0.438 & 3.229 & 2.5 & 110 & 0.58 & 83 & 27 \\
\hline & 17.5 & 0.5 & 3.229 & 2.5 & 110 & 0.58 & 83 & 27 \\
\hline & 20 & 0.313 & 3.229 & 2.5 & 110 & 0.58 & 125 & 25 \\
\hline & 20 & 0.375 & 3.229 & 2.5 & 110 & 0.58 & 115 & 30 \\
\hline & 20 & 0.438 & 3.229 & 2.5 & 110 & 0.58 & 102 & 28 \\
\hline & 20 & 0.5 & 3.229 & 2.5 & 110 & 0.58 & 102 & 28 \\
\hline & 17.5 & 0.313 & 3.229 & 2.5 & 130 & 0.64 & 70 & 25 \\
\hline & 17.5 & 0.375 & 3.229 & 2.5 & 130 & 0.64 & 63 & 30 \\
\hline & 17.5 & 0.438 & 3.229 & 2.5 & 130 & 0.64 & 63 & 28 \\
\hline & 17.5 & 0.5 & 3.229 & 2.5 & 130 & 0.64 & 63 & 28 \\
\hline & 20 & 0.313 & 3.229 & 2.5 & 130 & 0.64 & 80 & 25 \\
\hline & 20 & 0.375 & 3.229 & 2.5 & 130 & 0.64 & 75 & 30 \\
\hline & 20 & 0.438 & 3.229 & 2.5 & 130 & 0.64 & 73 & 28 \\
\hline & 20 & 0.5 & 3.229 & 2.5 & 130 & 0.64 & 73 & 28 \\
\hline & 17.5 & 0.313 & 3.229 & 2.5 & 140 & 0.7 & 62 & 25 \\
\hline & 17.5 & 0.375 & 3.229 & 2.5 & 140 & 0.7 & 57 & 30 \\
\hline & 17.5 & 0.438 & 3.229 & 2.5 & 140 & 0.7 & 57 & 28 \\
\hline
\end{tabular}


Table 1 (continued)

\begin{tabular}{|c|c|c|c|c|c|c|c|c|}
\hline \multirow[t]{2}{*}{ References } & \multicolumn{6}{|c|}{ Input variables } & \multicolumn{2}{|l|}{ Output } \\
\hline & $\begin{array}{l}\text { Excavation } \\
\text { Depth, H } \\
\text { (m) }\end{array}$ & $\begin{array}{l}\text { Length } \\
\text { to width } \\
\text { ratio of } \\
\text { excavation } \\
\text { L/B }\end{array}$ & $\begin{array}{l}\text { Ratio of } \\
\text { El wall/ } \\
\text { El of } \\
\text { brace }\end{array}$ & $\begin{array}{l}\text { Vertical } \\
\text { Spacing } \\
\text { between } \\
\text { bracing, S } \\
\text { (m) }\end{array}$ & $\begin{array}{l}\text { Undrained } \\
\text { Shear } \\
\text { strength } \\
\text { of clay, Su } \\
\left(\mathrm{kN} / \mathrm{m}^{2}\right)\end{array}$ & $\begin{array}{l}\text { Coefficient } \\
\text { of earth } \\
\text { pressure at } \\
\text { rest, Ko }\end{array}$ & $\begin{array}{l}\text { Maximum } \\
\text { deflection, } \\
\delta_{H}(\mathrm{~mm})\end{array}$ & $\begin{array}{l}\text { Position of } \\
\text { maximum } \\
\text { deflection, } \\
\text { (m) }\end{array}$ \\
\hline & 17.5 & 0.5 & 3.229 & 2.5 & 140 & 0.7 & 57 & 28 \\
\hline & 20 & 0.313 & 3.229 & 2.5 & 140 & 0.7 & 73 & 25 \\
\hline & 20 & 0.375 & 3.229 & 2.5 & 140 & 0.7 & 66 & 28 \\
\hline & 20 & 0.438 & 3.229 & 2.5 & 140 & 0.7 & 66 & 28 \\
\hline & 20 & 0.5 & 3.229 & 2.5 & 140 & 0.7 & 66 & 28 \\
\hline & 5 & 0.625 & 3.229 & 2.5 & 80 & 0.53 & 16 & 12 \\
\hline & 5 & 0.625 & 0.172 & 2.5 & 80 & 0.53 & 27 & 8 \\
\hline & 5 & 0.625 & 0.113 & 2.5 & 80 & 0.53 & 30 & 8 \\
\hline & 5 & 0.625 & 0.043 & 2.5 & 80 & 0.53 & 34 & 7 \\
\hline & 5 & 0.625 & 0.024 & 2.5 & 80 & 0.53 & 37 & 6.5 \\
\hline & 5 & 0.625 & 0.01 & 2.5 & 80 & 0.53 & 40 & 6 \\
\hline & 7.5 & 0.625 & 3.229 & 2.5 & 80 & 0.53 & 25 & 15 \\
\hline & 7.5 & 0.625 & 0.172 & 2.5 & 80 & 0.53 & 50 & 12 \\
\hline & 7.5 & 0.625 & 0.113 & 2.5 & 80 & 0.53 & 55 & 11 \\
\hline & 7.5 & 0.625 & 0.043 & 2.5 & 80 & 0.53 & 67 & 10.5 \\
\hline & 7.5 & 0.625 & 0.024 & 2.5 & 80 & 0.53 & 72 & 10 \\
\hline & 7.5 & 0.625 & 0.01 & 2.5 & 80 & 0.53 & 78 & 9.5 \\
\hline & 10 & 0.625 & 3.229 & 2.5 & 80 & 0.53 & 45 & 20 \\
\hline & 10 & 0.625 & 0.172 & 2.5 & 80 & 0.53 & 81 & 14 \\
\hline & 10 & 0.625 & 0.113 & 2.5 & 80 & 0.53 & 88 & 13 \\
\hline & 10 & 0.625 & 0.043 & 2.5 & 80 & 0.53 & 105 & 12.5 \\
\hline & 10 & 0.625 & 0.024 & 2.5 & 80 & 0.53 & 120 & 12 \\
\hline & 10 & 0.625 & 0.01 & 2.5 & 80 & 0.53 & 162 & 12 \\
\hline & 12.5 & 0.625 & 3.229 & 2.5 & 80 & 0.53 & 72 & 25 \\
\hline & 12.5 & 0.625 & 0.172 & 2.5 & 80 & 0.53 & 122 & 17 \\
\hline & 12.5 & 0.625 & 0.113 & 2.5 & 80 & 0.53 & 138 & 16.5 \\
\hline & 12.5 & 0.625 & 0.043 & 2.5 & 80 & 0.53 & 21.4 & 16 \\
\hline
\end{tabular}

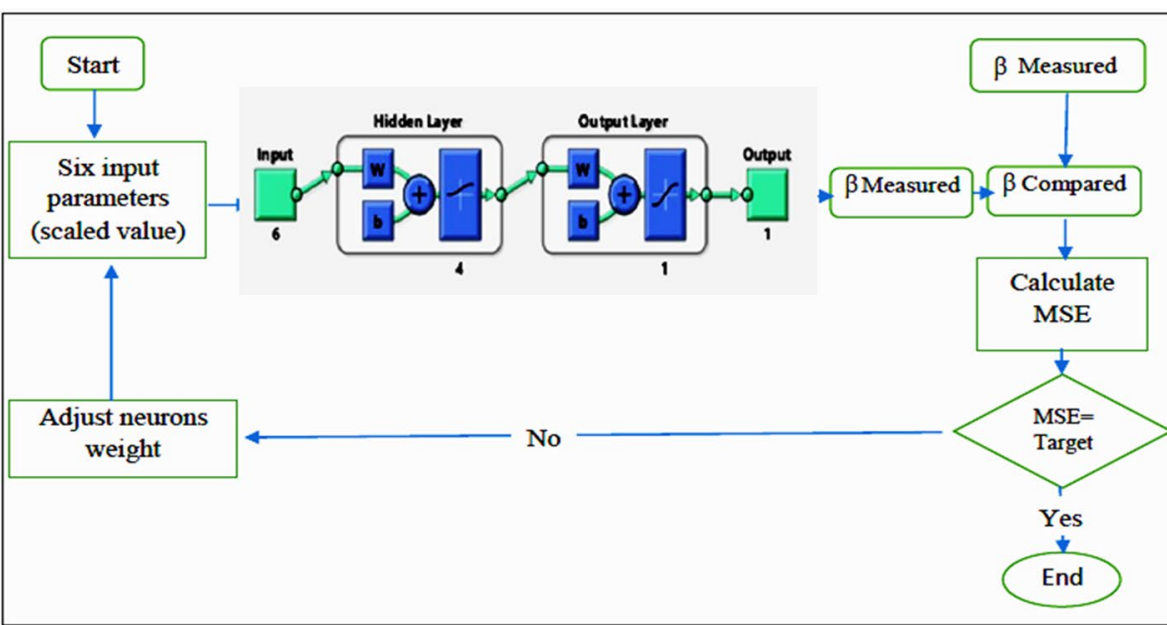

Fig. 2 Flow chart algorithm and structure of ANN for predicting max. Wall deflection 
Table 2 Statistics parameters of Input and Output data used in (ANN)

\begin{tabular}{|c|c|c|c|c|c|c|c|c|c|}
\hline \multirow{2}{*}{$\begin{array}{l}\text { Data } \\
\text { set }\end{array}$} & \multirow{2}{*}{$\begin{array}{l}\text { Statistical } \\
\text { parameters }\end{array}$} & \multicolumn{6}{|c|}{ Input variables } & \multicolumn{2}{|l|}{ Output } \\
\hline & & $\begin{array}{l}\text { Excavation } \\
\text { depth, H } \\
\text { (m) }\end{array}$ & $\begin{array}{l}\text { Length } \\
\text { to width } \\
\text { ratio of } \\
\text { excavation } \\
\text { L/B }\end{array}$ & $\begin{array}{l}\text { Ratio } \\
\text { of El } \\
\text { wall/ } \\
\text { El of } \\
\text { brace }\end{array}$ & $\begin{array}{l}\text { Vertical } \\
\text { Spacing } \\
\text { between } \\
\text { bracing, } \\
S(m)\end{array}$ & $\begin{array}{l}\text { Undrained } \\
\text { Shear } \\
\text { strength } \\
\text { of clay, Su } \\
\left(\mathrm{kN} / \mathrm{m}^{2}\right)\end{array}$ & $\begin{array}{l}\text { Coefficient } \\
\text { of earth } \\
\text { pressure } \\
\text { at rest, Ko }\end{array}$ & $\begin{array}{l}\text { Maximum } \\
\text { deflection, } \\
\delta \mathrm{H}(\mathrm{mm})\end{array}$ & $\begin{array}{l}\text { Position of } \\
\text { maximum } \\
\text { deflection, } \\
\text { (m) }\end{array}$ \\
\hline \multirow{5}{*}{$\begin{array}{l}\text { All Data } \\
n=169\end{array}$} & Maximum & 20.0 & 2.7 & 38.7 & 6 & 140.0 & 0.70 & 300.0 & 95.0 \\
\hline & Minimum & 2.2 & 0.2 & 0.010 & 0 & 15.0 & 0.53 & 1.0 & 0.0 \\
\hline & Mean & 11.3 & 0.6 & 5.7 & 2.4 & 81.8 & 0.6 & 65.4 & 19.5 \\
\hline & Std.dv & 5.6 & 0.3 & 8.2 & 0.8 & 30.7 & 0.0 & 46.5 & 11.8 \\
\hline & Range & 17.8 & 2.5 & 38.7 & 6.0 & 125.0 & 0.2 & 299.0 & 95.0 \\
\hline
\end{tabular}

ANNs with 1 to 12 hidden layer nodes are trained, to determine the optimum network geometry.

\section{Training of data}

Table 2 summarizes statistical parameters of variables used to construct and validate the network and their histograms are shown in Fig. 3. The retaining structures are formed of either stiff or flexible walls with Excavation depth up to $20 \mathrm{~m}$ and maximum width up to $80 \mathrm{~m}$ was considered.

Figures 4 and 5 indicate the variation of the average of Mean Squared Error (MSE) with the number of hidden neurons of the networks for the maximum deflection and its position respectively. The difference in (MSE) is relatively small after hidden neuron no. 4 and hidden neuron no. 3 for the maximum deflection and its position respectively. Figure 4 shows that the networks for max. deflection and its position with 4 and 3 hidden layer node respectively. It is considered optimal, as its prediction error (MSE) is not far from the network with 12 hidden layer nodes coupled with smaller number of connection weights and with high coefficient of correlation as shown in Fig. 5

Figures 6 and 7 show a comparison between the measured and predicted value of the maximum deflection and it's position respectively. It shows a good agreement between the measured and predicted values.

\section{Sensitivity analysis of the ANN model inputs}

Garson [4] proposed an innovative and simple technique that is used to explain the relative significance of the input variables by examining the connection weights of the trained network. For a network with 4 hidden nodes, the technique involves a process of partitioning the hidden output connection weights into components associated with each input node.

The results indicate that the ratio of the EI wall/EI of brace has the most considerable effect on the predicted maximum deflection followed by Ko, L/B, H, Su, and hs respectively. The results are presented in Fig. 8a. Similarly, the results indicate that $\mathrm{Su}$ and Ko have the most significant effect on the predicted position of maximum deflection followed by L/B, H, hs, and EI/Ks, the results are presented in Fig. 8b. 


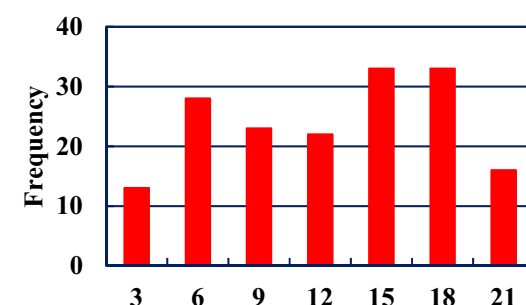

Excavation Depth, H (m)
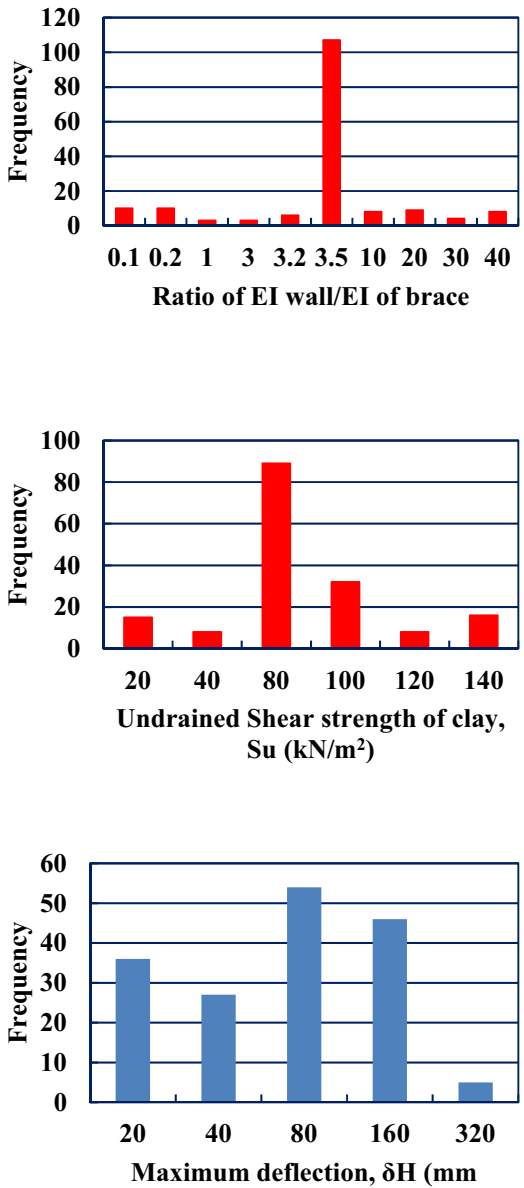

Fig. 3 Histograms of the data used
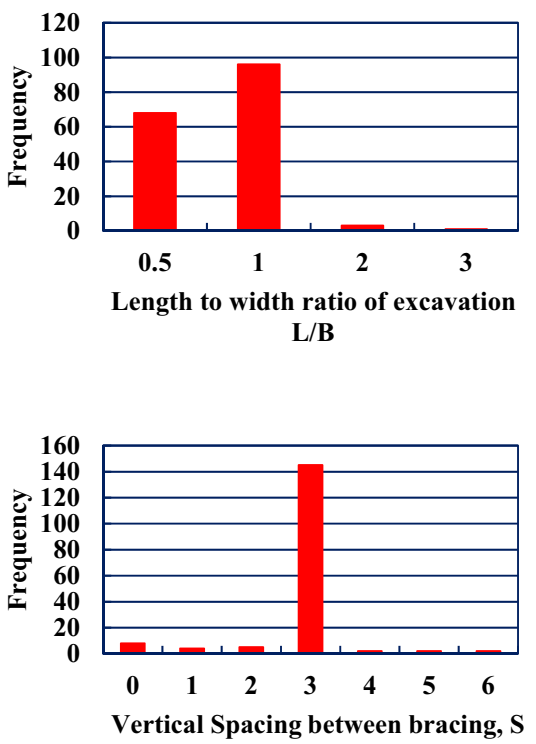

(m)
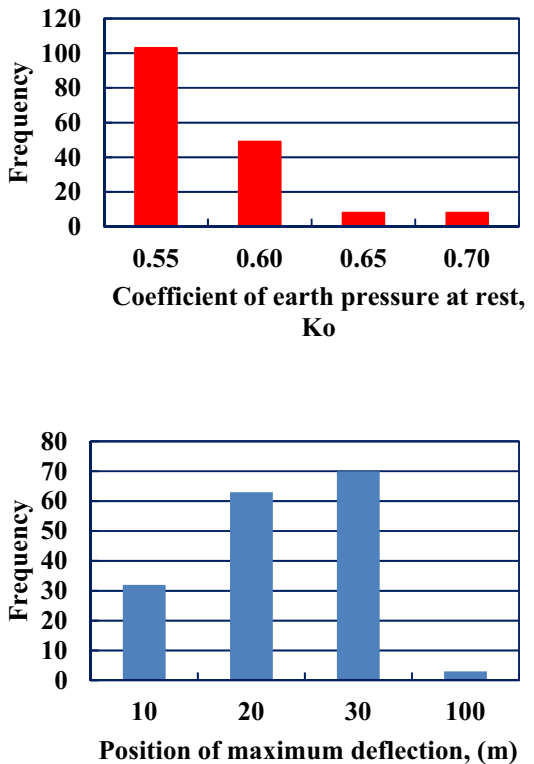

\section{Parametric study}

Parametric studies were carried out to estimate whether the model has convenient trends, to confirm the validation of the trained network and the generalization ability of the model. the first five input variables (i.e. (L/B), EI of wall/stiffness of braced, Vertical distance of bracing, Undrained shear strength of soil $(\mathrm{Su})$, coefficient of Earth pressure at rest $\left(\mathrm{K}_{\mathrm{o}}\right)$ ), except one, are fixed to their mean values used for training. The increasing values of artificial ranging (10-20\%) of the total range between the minimum and maximum values. Figure $9 \mathrm{a}-\mathrm{d}$ reveal that the general trend of the maximum wall deflection is to increase with an increase in excavation depth, increasing (L/B), (EI wall/EI of bracing) 

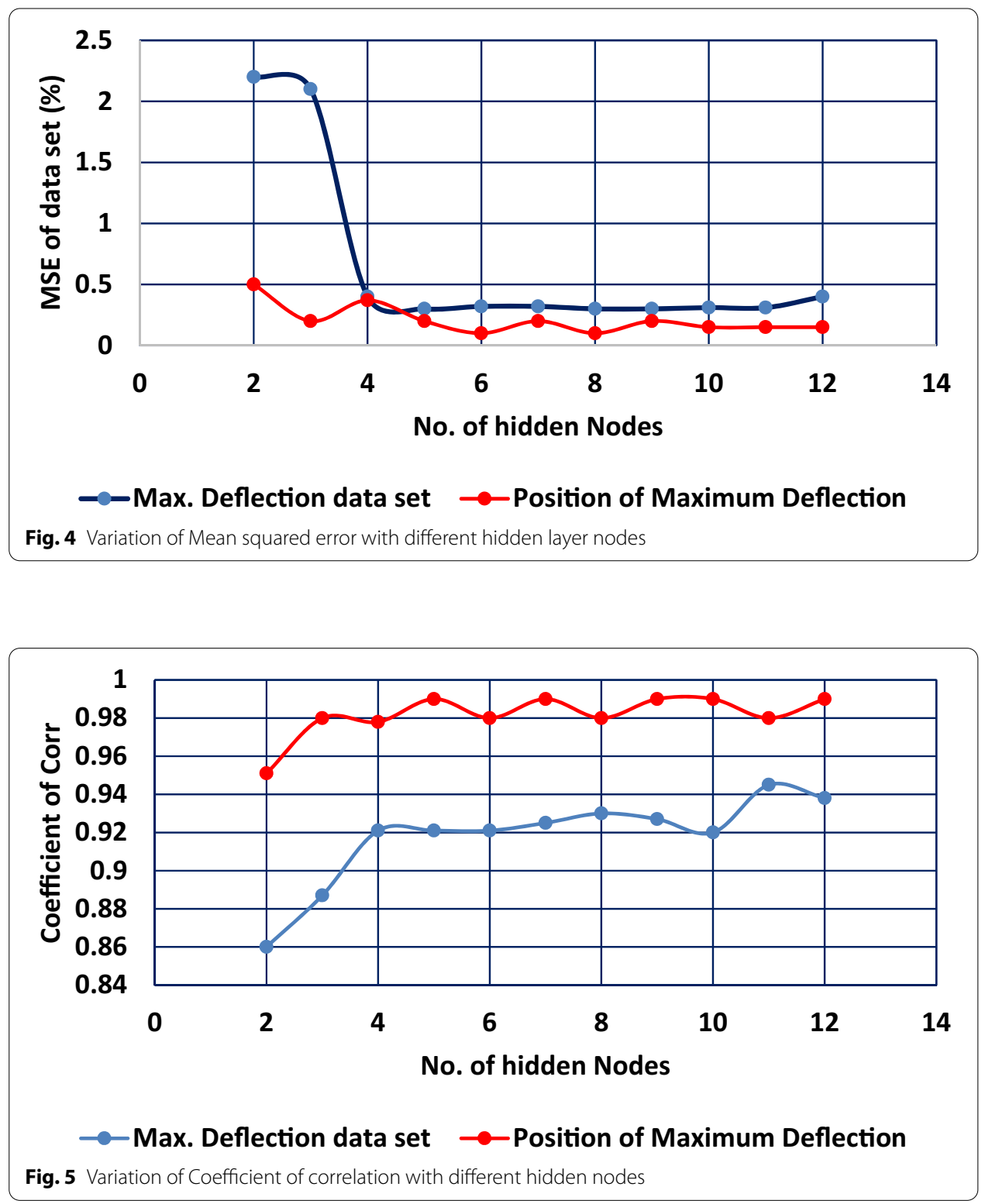

ratios and vertical distance of bracing respectively as expected. Figure 9e shows that the changes in max. wall deflections were less significant for Su greater than 75 MPA, and increase with the increasing coefficient of lateral earth pressure as shown in Fig. $9 f$.

The general trend for the position of max. Wall deflection was revealed in Fig. 10a$\mathrm{f}$, it increases with the increase in excavation depth, while decreases with $(\mathrm{L} / \mathrm{B})$ ratios, (EI/stiffness of bracing) ratios, and the vertical distance of bracing $\left(\mathrm{h}_{\mathrm{s}}\right)$, on the other hand the position of max. deflection increases with the increase in shear strength of the soil $(\mathrm{Su})$ up to $60 \mathrm{kN} / \mathrm{m}^{2}$ then decreases, and the same trend observed with the coefficient of lateral earth pressure $\left(\mathrm{K}_{\mathrm{o}}\right)$. Consequently, the trained neural network model was approved to give reasonable and appropriate relationships between the diverse input variables. The developed ANN has shown satisfactory performance. 

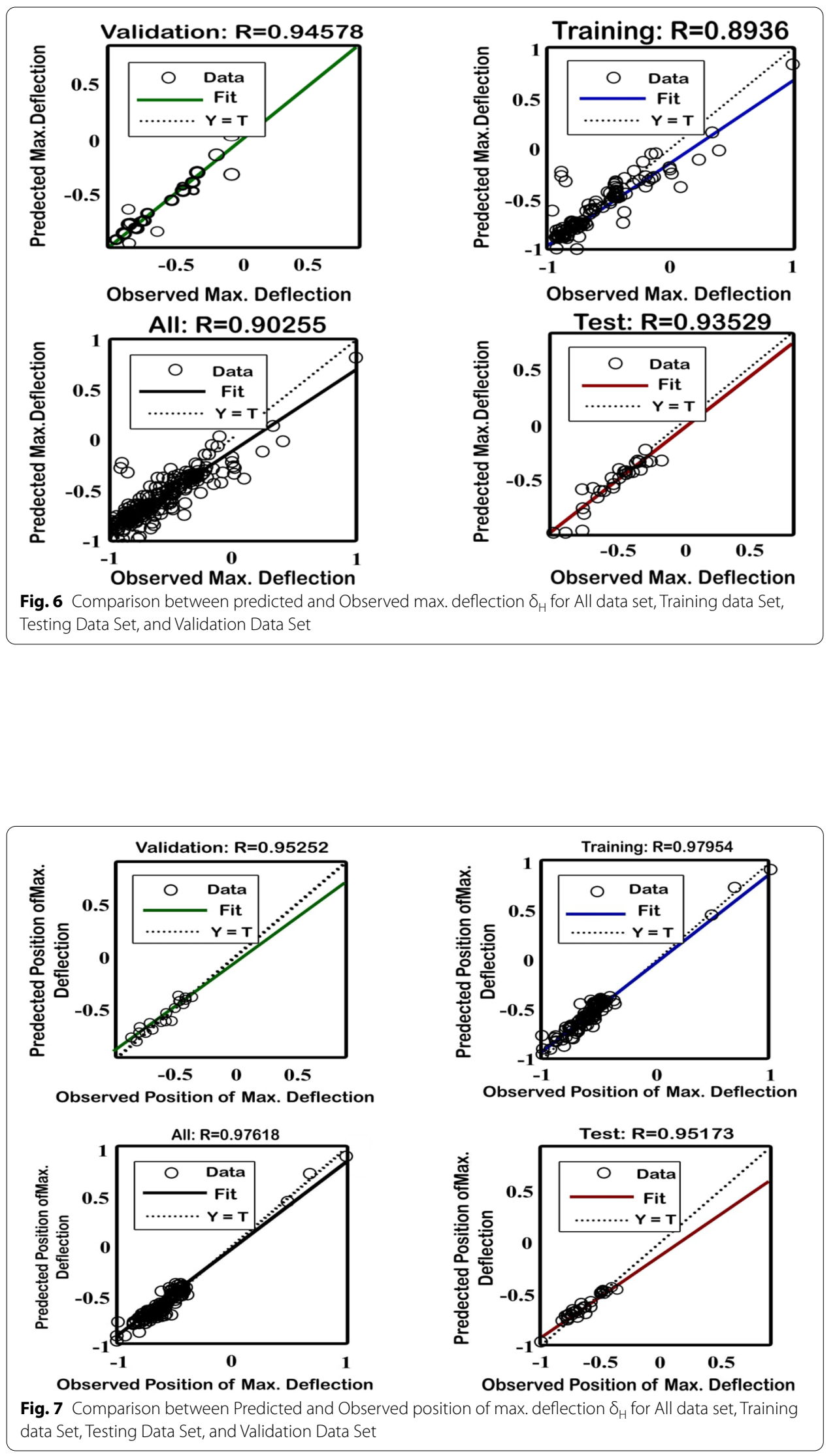


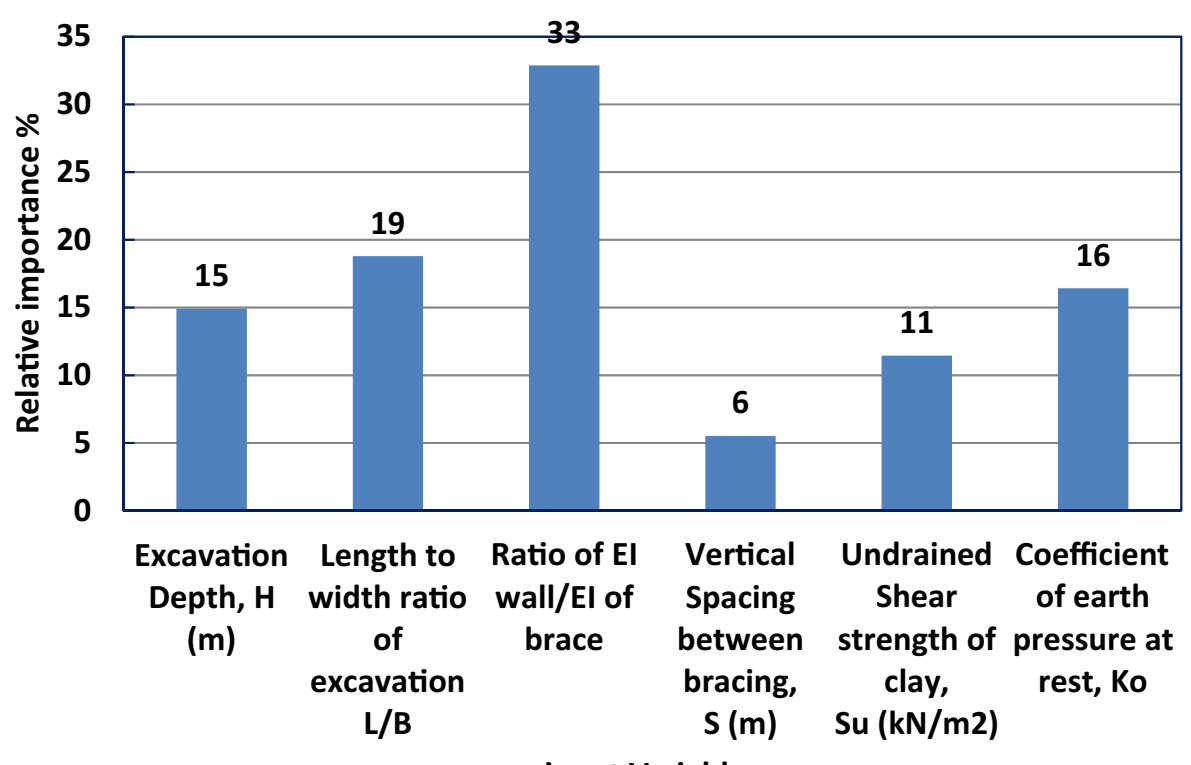

(a)

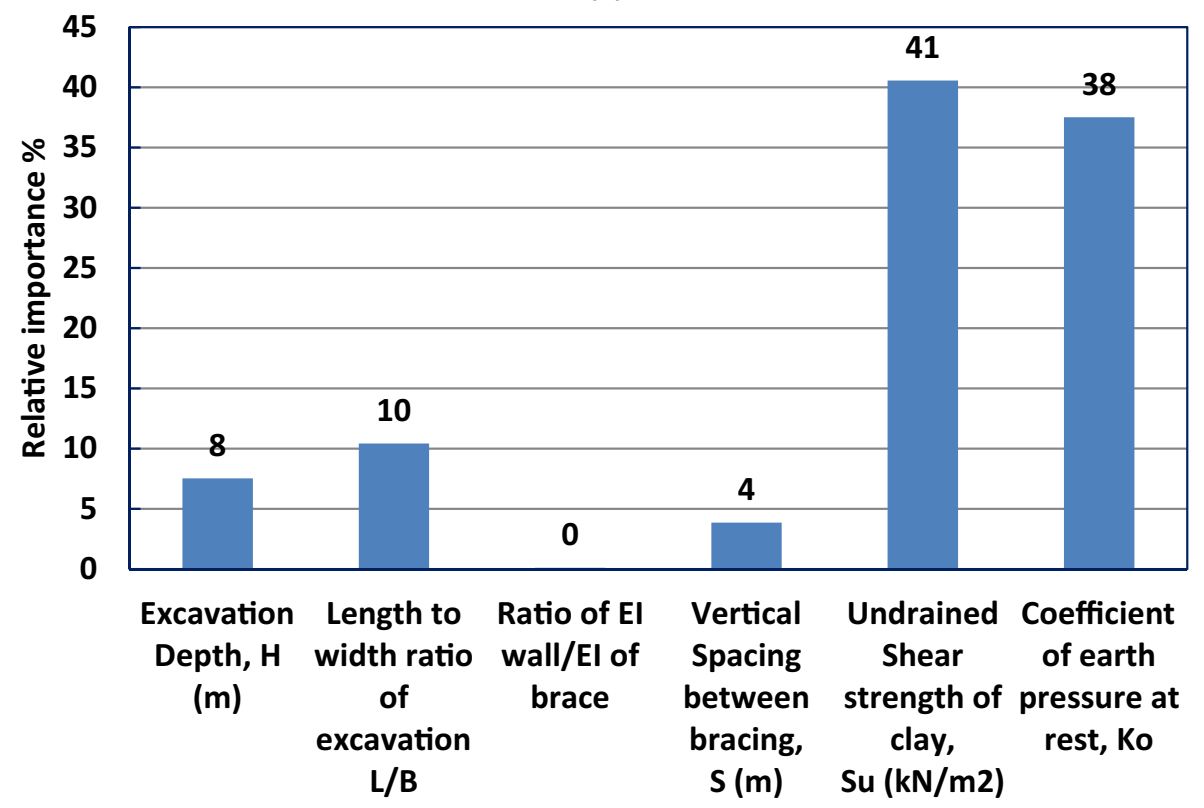

(b)

Fig. 8 The relative importance of input variable for the value of a max. deflection, $\mathbf{b}$ Position of max. deflection

A set of design charts are developed. It's carried out by using synthetic input values within the ranges of data shown in Table 2. Figure 11 is an illustrative sample of the design charts obtained for $L / B=1, K_{o}=0.53, h_{s}=2.5 \mathrm{~m}$, the increasing trend of varying with excavation depth $(\mathrm{H})$ for all cases, maximum deflection increases as the excavation depth increases and as expected. 


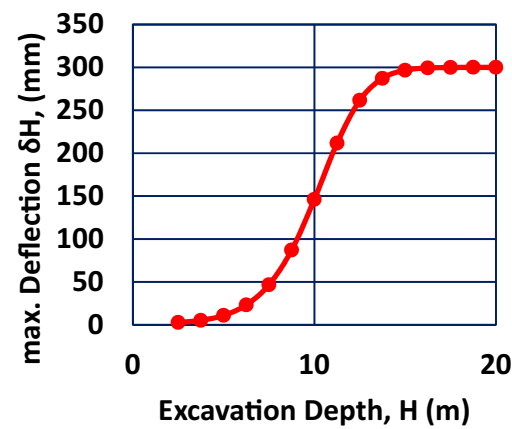

(a)

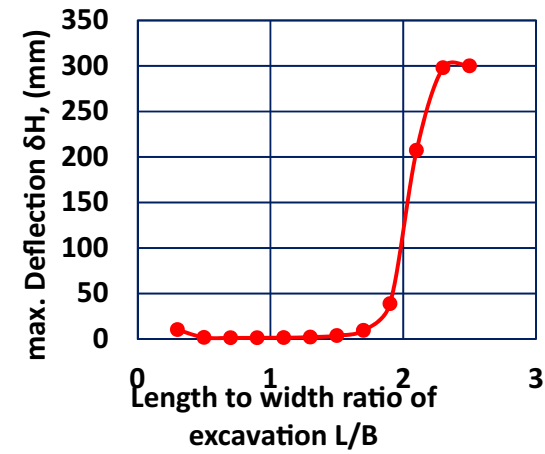

(b)

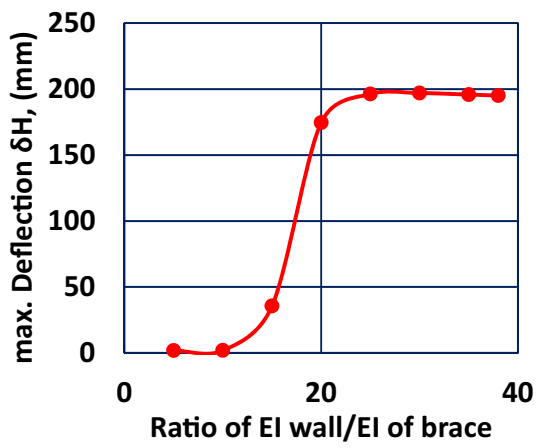

(c)

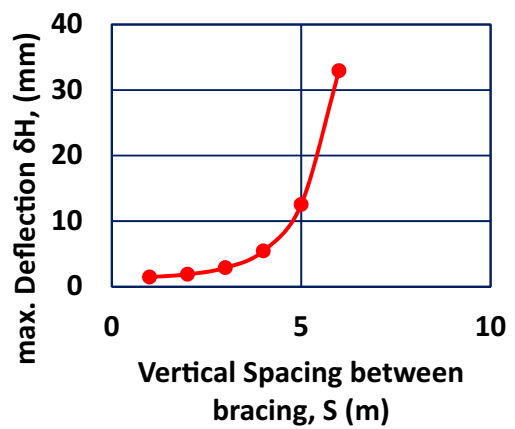

(d)

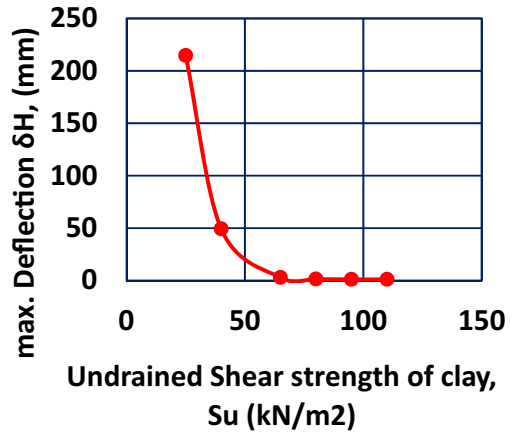

(e)

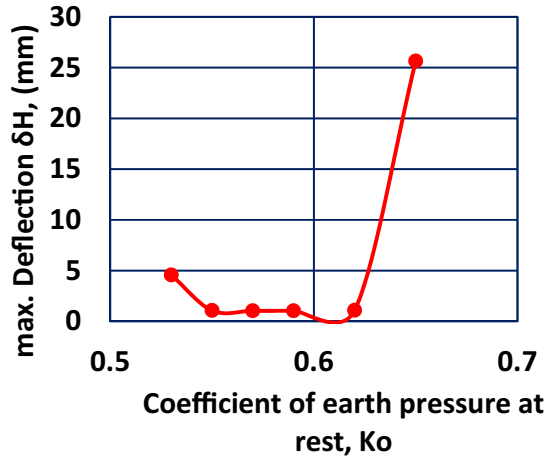

(f)

Fig. 9 Result of a parametric study of (ANN) model for the max. excavation under different, a excavation depth, $(H)$, b length to width ratio, $(L / B)$, c ratio of El wall/El of bracing, $\mathbf{d}$ vertical distance of bracing between spacing, e undrained shear strength, Su, f coefficient of lateral earth pressure at rest Ko

On the other hand, the robustness of the (ANN) for the position of max. deflection can be determined by examining with input values within the ranges of data shown in Table 2, the sample of design chart obtained for $\mathrm{L} / \mathrm{B}=1, \mathrm{Ko}=0.53$, hs $=2.5 \mathrm{~m}$, EI wall/EI brace $=7$, It was found that the trends of the design charts are in good agreement with what one would expect as shown in Fig. 12

\section{Summary and conclusions}

In this study, a neural network-based procedure to predict maximum wall excavation during excavation has been proposed. Based on a large number of case studies generated by observation techniques and FEM, the ANN model is developed to predict the 


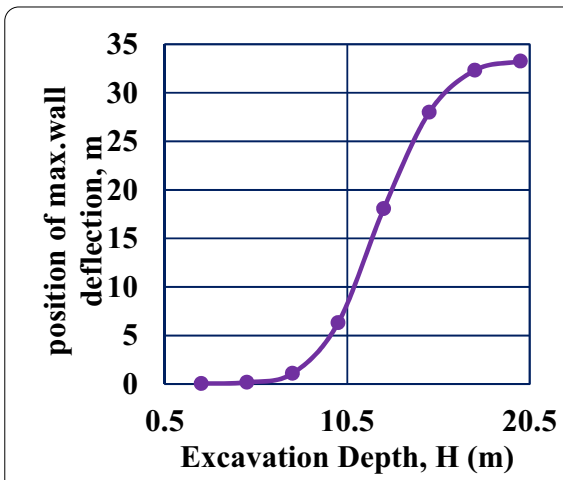

(a)

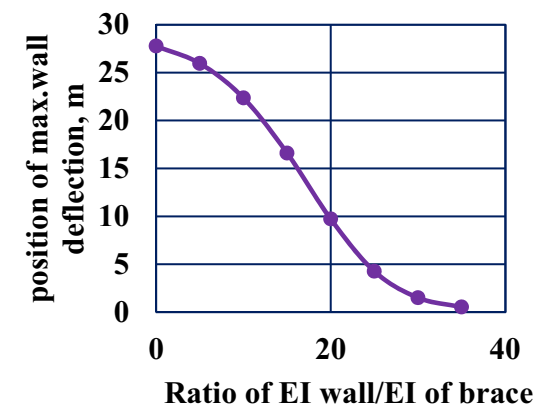

(c)

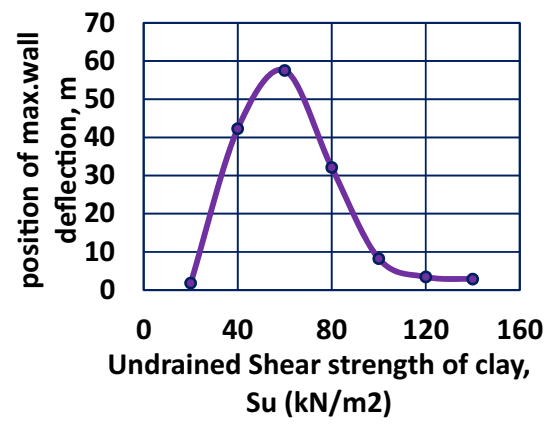

(e)

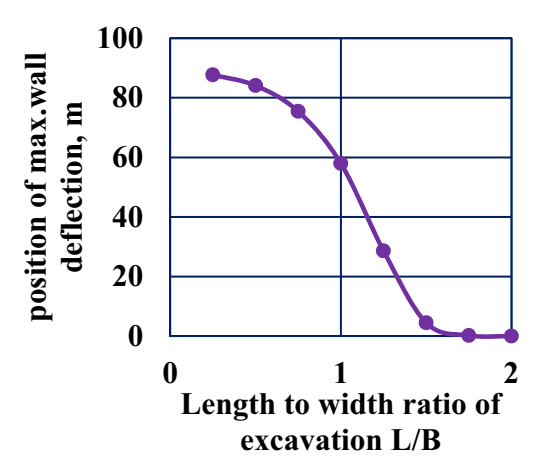

(b)

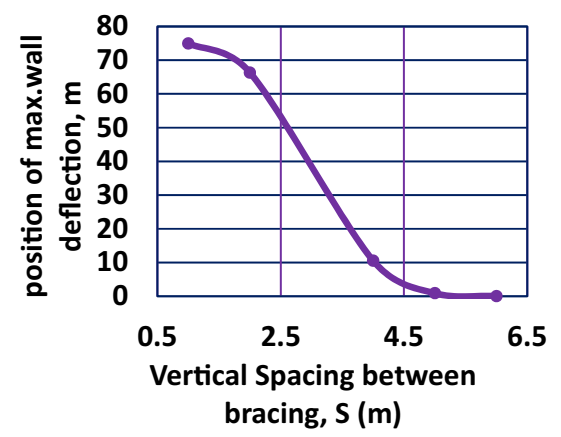

(d)

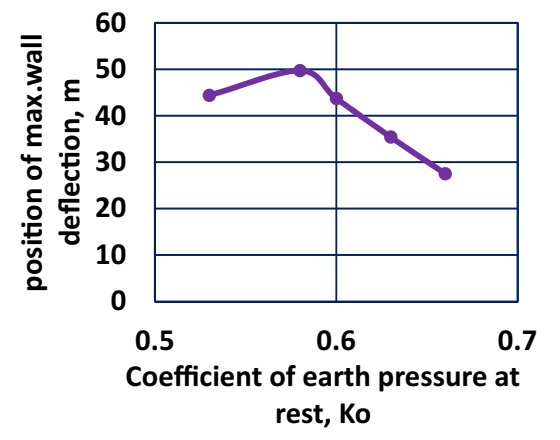

(f)

Fig. 10 Result of a parametric study of (ANN) model for the position of max. excavation under different, a excavation depth, $(\mathrm{H})$, b length to width ratio, $(\mathrm{L} / \mathrm{B})$, c ratio of El wall/El of bracing, $\mathbf{d}$ vertical distance of bracing between spacing, e undrained shear strength, Su, $\mathbf{f}$ coefficient of lateral earth pressure at rest Ko

max. wall deflection induced by excavation. Six input variables are adopted to build the ANN model; they are excavation depth, system stiffness, the vertical distance between bracing, excavation width/wall length ratio, shear strength, and the lateral earth pressure coefficient. The effect of using a various number of a hidden layer on the ANN model results was investigated. The relative importance of the factors that affect maximum lateral deflection and its position was achieved by sensitivity analysis. The typical behavior of braced excavation in soft to medium clay is confirmed by the results of the extensive assessment of the ANN model developed.

The results showed that the sensitivity analysis indicates that the system stiffness has the most significant effect on the maximum wall deflection. The soil properties 


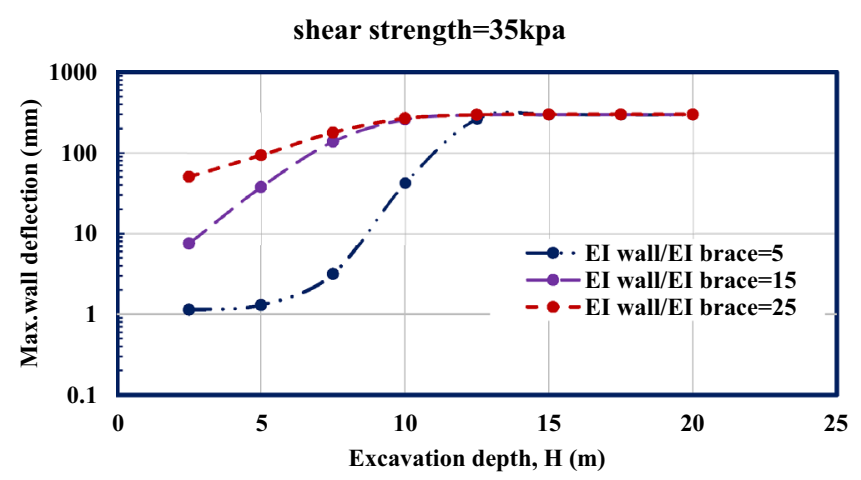

(a)

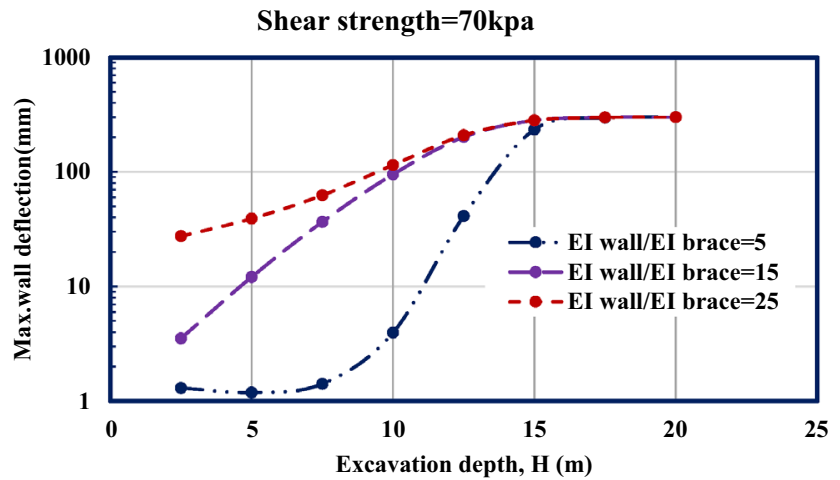

(b)

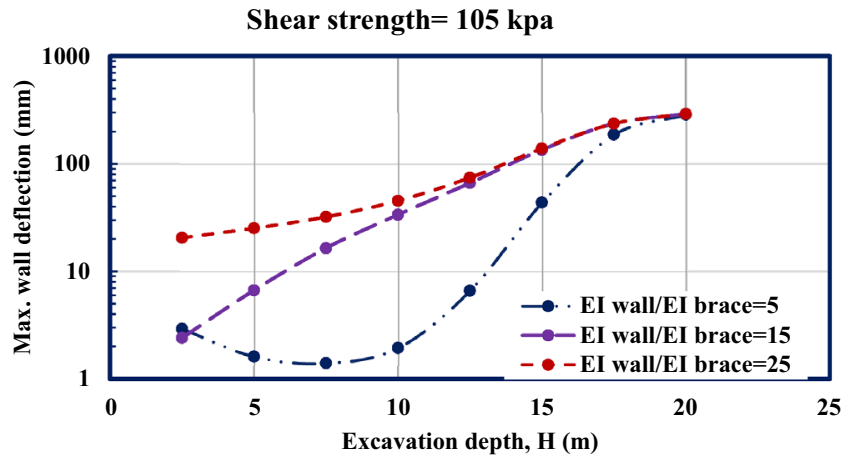

(c)

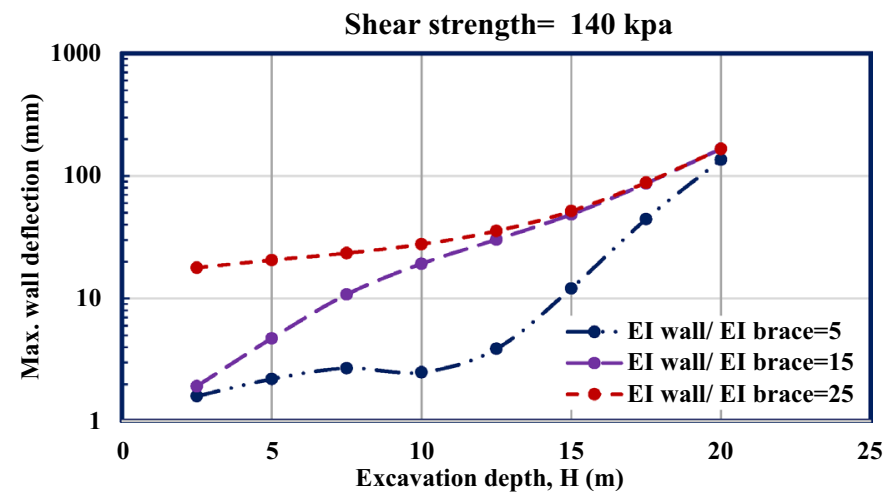

(d)

Fig. 11 Maximum wall deflections predicted by the $A N N$ versus variation excavation depth, for $L / B=1$, $\mathrm{K}_{\mathrm{o}}=0.53, \mathrm{~h}_{\mathrm{s}}=2.5 \mathrm{~m}$ 


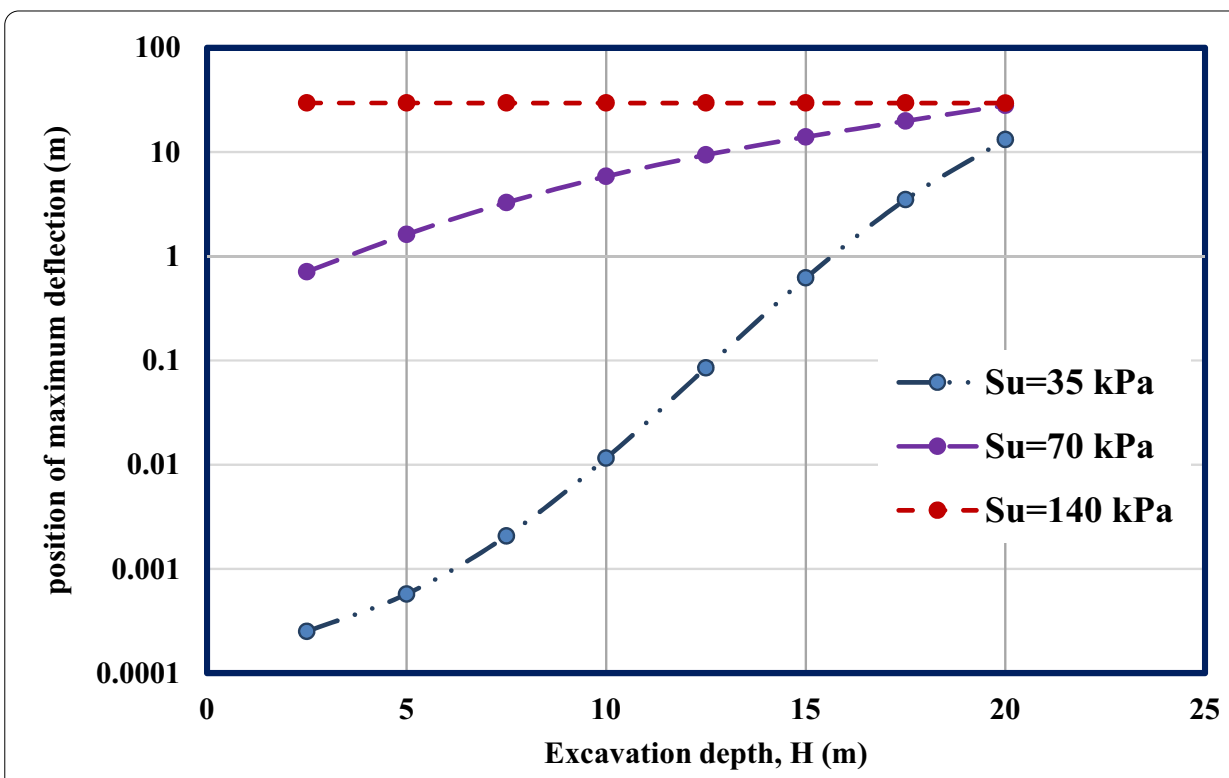

Fig. 12 Position of maximum wall deflections predicted by the ANN versus variation excavation depth, for $\mathrm{L} / \mathrm{B}=1, \mathrm{Ko}=0.53, \mathrm{hs}=2.5 \mathrm{~m}$, El wall/El brace $=7$

have the most significant effects on the position, alternately the system stiffness and the vertical distance between bracing were found to have minor effects compared to the soil properties depending on the position of max. wall deflection.

The ANN developed has shown to be able to predict the wall deflections satisfactorily as showed by the results of the validation. Degree of accuracy was acceptable $(R=0.922$, MSE $=0.4 \%$, and the number of optimum hidden layer nodes was found to be four) for predicted maximum wall deflection, and $(\mathrm{R}=0.978, \mathrm{MSE}=0.22 \%$, and the number hidden layer nodes was found to be three hidden layer nodes) for predicted position of maximum wall deflection. The developed ANN trend to improved results based on the mean of case histories.

Acknowledgements

The authors would like to thank the University of Anbar, college of engineering for their support to carry the study

Authors' contributions

All authors read and approved the final manuscript.

\section{Declarations}

\section{Competing interests}

The authors declare that they have no competing interests.

\section{Author details}

${ }^{1}$ Civil Engineering, University of Anbar, Anbar, Iraq. ${ }^{2}$ Directorate of Engineering \& Technical Affairs, Ministry of Youth and Sports, Anbar, Iraq.

Received: 8 July 2020 Accepted: 24 May 2021

Published online: 07 October 2021 
References

1. Choo CY, Erwin OH (2016) Modelling ground response for deep excavation in soft ground. Int J Geomate 11(26):2633-2642

2. Dayhoff JE (1990) Neural network architectures: an introduction. Van Nostrand Reinhold Press, New York

3. Flood I, Kartam N (1994) Neural networks in civil engineering. I: principles and understanding. J Comput Civ Eng 8(2):131-148. https://doi.org/10.1061/(ASCE)0887-3801(1994)8:2(131)

4. Garson GD (1991) Interpreting neural-network connection weights. Al Expert 6(4):47-51

5. Hagan MT, Demuth HB, Beale MH, De Jess O (2014) Neural network design, 2nd edn. Martin Hagan, USA

6. Hashash YMA, Marulanda C, Ghaboussi J, Jung S (2006) A novel approach to integration of numerical modeling and field observations for deep excavations. J Geotech Geoenviron Eng 123(8):1019-31. https://doi.org/10.1061/(ASCE) 1090-0241(2006)132:8(1019)

7. Hashash YMA, Whittle AJ (1996) Ground movement prediction for deep excavations in soft clay. J Geotech Eng 122(6):474-486. https://doi.org/10.1061/(ASCE)0733-9410(1996)122:6(474)

8. Jan JC, Hung SL, Chi SY, Chern JC (2002) Neural network forecast model in deep excavation. J Comput Civ Eng 16:59-65. https://doi.org/10.1061/(ASCE)0887-3801(2002)16:1(59)

9. Jen LC (1998) The design and performance of deep excavation in clay. Ph.D. dissertation. Cambridge, MA, USA: Department of Civil and Environmental Engineering, MIT. http://hdl.handle.net/1721.1/10017

10. Kartam N, Flood I, Garrett JH (1997) Artificial neural networks for civil engineers: fundamentals and applications. ASCE, New York

11. Kung GTC, Hsiao ECL, Schuster M, Juang CH (2007) A neural network approach to estimating deflection of diaphragm walls caused by excavation in clays. Comput Geotech 34(5):385-396. https://doi.org/10.1016/j.compgeo. 2007.05.007

12. Lam SY, Haigh SK, Bolton MD (2014) Understanding ground deformation mechanisms for multi-propped excavation in soft clay. Soils Found 2014(54):296-312. https://doi.org/10.1016/j.sandf.2014.04.005

13. Lam SY (2010) Ground movements due to excavation in clay: physical \& analytical models. PhD dissertation, University of Cambridge

14. Nicholson DP (1987) The design and performance of retaining walls at Newton Station. In: Proceedings of the Singapore mass rapid transit conference, 6-9 April, Singapore, pp 147-154

15. Peck RB (1969) Deep excavations and tunneling in soft ground. In: Proc., 7th Int. Conf. Soil Mechanics and Foundation Engineering, Univ. Nacional Autonoma de Mexico Instituto de Ingenira, Mexico City, pp 225-290

16. Whittle AJ, Hashash YMA, Whitman RV (1993) Analysis of deep excavation in Boston. J Geotech Eng 119(1):69-90. https://doi.org/10.1061/(ASCE)0733-9410(1993)119:1(69)

17. Yildiz E (2003) Lateral pressures on rigid retaining walls: a neural network approach. M.Sc. thesis, the Graduate School of Applied Sciences, Middle East Technical University

\section{Publisher's Note}

Springer Nature remains neutral with regard to jurisdictional claims in published maps and institutional affiliations.

\section{Submit your manuscript to a SpringerOpen ${ }^{\circ}$ journal and benefit from:}

Convenient online submission

- Rigorous peer review

- Open access: articles freely available online

- High visibility within the field

Retaining the copyright to your article

Submit your next manuscript at $\boldsymbol{\Delta}$ springeropen.com 